\title{
Another Successful E-MRS Meeting is Held in Strasbourg
}

\author{
ICAM '91 Shares Venue
}

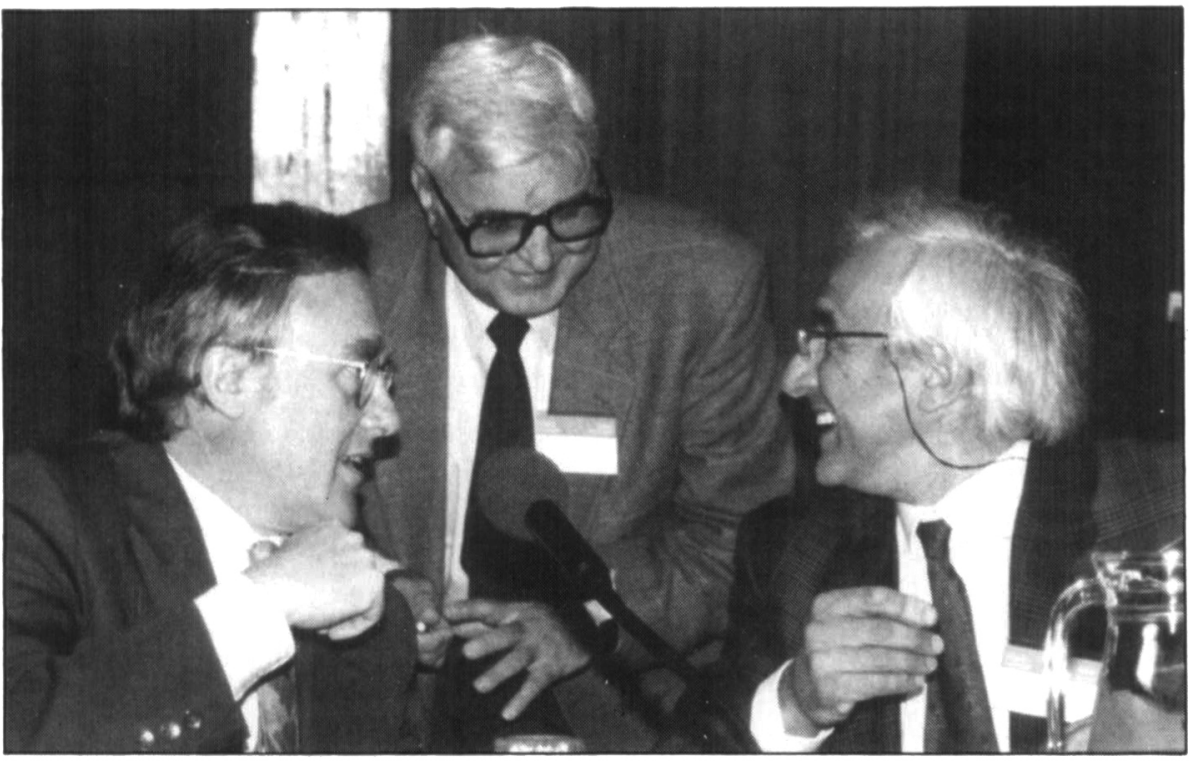

J.P. Massué, Council of Europe, (left) confers with ICAM Chair M. Balkanski, University of Pierre and Marie Curie, and E-MRS President G.G. Bentini, CNR, Bologna, Italy.

The European Materials Research Society held yet another successful spring meeting during the week of May 27, 1991 at the facilities of the Council of Europe and the European Parliament in Strasbourg, France. Sharing the Strasbourg venue was the 1991 International Conference on Advanced Materials (ICAM).

The E-MRS meeting featured five symposia: New Materials Physics and Technologies for Micronic Integrated Sensors, High Energy Ion Implantation, Ion Beam Synthesis of Compound and Elemental Layers, Laser Surface Processing and Characterization, and Nuclear Methods in Semiconductor Physics.

ICAM '91, the second in a series of international conferences sponsored under the auspices of the International Union of $\mathrm{Ma}$ terials Research Societies, featured four symposia: High Temperature Superconductor Thin Films, Solid State Ionics, Nonstoichiometry in Semiconductors, and Composite Materials. The first such meeting was held in Tokyo, Japan in 1988.

In addition to these nine symposia, a plenary session for all participants of the joint conference was held on Tuesday morning, May 28. Plenary speakers addressed topics on high temperature super- conductivity, stoichiometry control of compound semiconductor crystals, and research on surfaces and interfaces. During the plenary session, several graduate student awards were presented to symposium participants.

With nearly 800 participants, the meeting was well attended. According to then president of E-MRS, G.G. Bentini (Bologna, Italy), this number is just the appropriate size for the Council of Europe facilities which housed the meeting.

During a business meeting at the May conference, the E-MRS Board of Delegates elected new members of its Executive Committee, the Society's operating body. (See related article in the July MRS Bulletin, p. 40.) Also attending the E-MRS business meeting were representatives of Eastern European countries interested in E-MRS membership.

Some technical highlights of the symposia follow. For more details, consult the proceedings, which will be published by Elsevier.

\section{High Temperature Superconductor Thin Films}

Chairs: L. Correm (CNR-Istituto LAMEL,
Italy), T. Kawai (Osakn University, Japan), T. Venkatesan (University of Manyland, U.S.A.).

This symposium focused on interdisciplinary aspects of superconducting oxide thin films, covering fundamental properties to applications. Over 40 contributed papers, 110 posters, and 21 invited talks were presented during this five-day meeting, averaging 130 attendees. Most papers dealt with the 1-2-3 system of $\mathrm{Y}-\mathrm{Ba}-\mathrm{Cu}-\mathrm{O}$ and related compounds. However, interesting results for the $\mathrm{Bi}$ system were also presented.

The plenary session featured three presentations: E.N. Kaufmann (Argonne National Laboratory), H. Kamimura (Univ. of Tokyo), and W. Bückel (Karlsruhe) discussed theoretical aspects and current problems in high $\mathrm{T}_{\mathrm{c}}$ superconductor materials.

The meeting was divided into 15 oral sessions. To develop a better understanding of the relationships between microstructure and properties, four sessions were devoted to physical properties and two to structural characteristics. Superconductivity and two-dimensional transport behavior in $Y$ $\mathrm{Ba}-\mathrm{Cu}-\mathrm{O}$ and $\mathrm{Bi}-\mathrm{Sr}-\mathrm{Ca}-\mathrm{Cu}-\mathrm{O}$ thin films were widely discussed, as were optical, magnetic, and microwave properties. Film growth mechanisms were studied by various techniques, including RBS-channeling, $x$-ray diffraction, and highresolution TEM, the latter a powerful technique to study layer-by-layer epitaxy.

The main deposition techniques covered were laser ablation, evaporation, sputtering, and chemical vapor deposition. Complete control over deposition conditions is required for growing complex structures and for compatibility with device fabrication technology. Considerable progress was reported for high-quality thin-film preparation by CVD and organometallic CVD.

The papers devoted to processing emphasized deposition of epitaxial and smooth films on large area substrates and control of film orientation. The importance of methods for film deposition on silicon was highlighted. Surface and interface studies, including growth and properties of yttria-stabilized zirconia buffer layers, were discussed in a session devoted to substrates and multilayers.

Two sessions on applications illustrated significant progress in the fabrication of microwave devices, IR detectors, SQUID 
magnetometers and optoelectronic devices. However, many challenging problems remain for junction-based technology.

Two well-attended poster sessions were held in parallel to the equipment exhibition, offering symposium participants an opportunity to interact with industry representatives.

Two awards were presented to graduate students for their work in the high $\mathrm{T}_{c}$ superconductor field: to A. Angurel (Univ. of Zaragoza, Spain) for a paper on diamagnetic properties of $\mathrm{YBa}_{2} \mathrm{Cu}_{3} \mathrm{O}_{7 \times}$ thin films and to $A$. Sacuto (Univ. of Pierre and Marie Curie, France), whose paper addressed the determination of the superconducting gap by Raman spectroscopy.

\section{Solid State Ionics}

Chairs: M. Balkanski (University of Pierre and Marie Curie, France), T. Takahashi (University of Nagoya, Japan), H. Tuller (Massachusetts Institute of Technology, U.S.A.).

The field of solid state ionics has grown rapidly over the past several decades, stimulated by both fundamental issues relating to transport and structure and by new applications in energy conversion and storage, sensors, electrochromic windows, and catalysis. The symposium on Solid State lonics addressed these issues in a series of 21 invited and 110 contributed papers.

In general, the sessions were concerned with identifying solids with higher ionic conductivities, lower operating temperatures, greater stability and with devices that lasted longer, responded faster, and could be fabricated thinner, with more flexibility, and at a lower cost. Many characterization methods, some new or refined, were introduced, including photoacoustic, IR, Raman, and EPR (electron paramagnetic resonance) spectroscopies, neutron and $x$-ray diffraction, XAFS ( $x$-ray absorption fine structure), and various electrochemical methods.

In the area of applications, S. Mennicke (ABB, Germany) described progress in the development of beta-alumina sodiumsulfur batteries for automotive use. Over $400,000 \mathrm{~km}$ of testing were reported without battery failures. Developments in using oxygen ion and proton conductors in fuel cells and electrolyzers were described by B.C.H. Steele (Imperial College, U.K.) and $\mathrm{H}$. Iwahara (Nagoya Univ., Japan). F.G.K. Baucke (Schott Glaswerke, Germany) reported on progress in electrochromics for smart windows and mirrors, while J. Livage (Univ. of Pierre and Marie Curie, France) described how some of these structures could be fabricated via sol- gel ionics. W. Weppner (Max-PlanckInstitut, Stuttgart, Germany) concentrated on new concepts in solid state electrochemical sensors which allow monitoring a wider range of chemical species.

In a session on new materials, J.B. Goodenough (Univ. of Texas, U.S.A.) described recent work on new oxide-ion electrolytes, and T. Minami (Univ. of Osaka Pref., Japan) reported on new $\mathrm{Cu}$ conducting glasses. B. Scrosati (Univ. of Rome, Italy) and D. Shriver and co-workers (Northwestern Univ., U.S.A.) discussed advances in conducting polymers in a session on polymeric electrolytes. Given the importance of mass transport in achieving optimum properties in the high temperature superconductors, several invited presentations were given on oxygen transport and defects in cuprates by $\mathrm{P}$. Barboux and co-workers (Univ. of Pierre and Marie Curie), J. Maier and co-workers (Max-PlanckInstitut, Stuttgart), and E. Opila and co-workers (MIT, U.S.A.).

In a session on transport, $\mathrm{T}$. Takahasi (Nagoya Univ., Japan) reviewed room temperature ionic conductors, C.R.A. Catlow (Royal Institution, U.K.) progress in computer modeling of defect and migration mechanism, and M. Ribes and co-workers (Univ. of Montpellier, France) relaxation processes in glasses. In a related session on structural effects, B.J. Wuensch (MIT, U.S.A.) correlated silver and copper transport, and structure in solids with simple anion packing. Deppe and co-workers (Univ. of Pierre and Marie Curie) examined structural effects in thioborate glasses.

Mixed ionic-electronic conductors were discussed by I. Riess (Technion, Israel), who described new methods for their analysis, and by I. Kosacki and co-workers (Univ. of Pierre and Marie Curie), who reported mixed conduction in $\mathrm{V}_{2} \mathrm{O}_{5}-\mathrm{LiBO}_{2}$ glasses. In a session on insertion compounds, C. Delmas (CNRS, Bordeaux, France) described the electrochemical behavior of nickel hydroxide electrodes.

Overall, one was left with the feeling that the field remained vibrant, that major applications were finally taking hold, and that future meetings would continue to provide fertile grounds for discussion and speculation.

\section{Nonstoichiometry in Semiconductors}

K.J. Bachmann (North Carolina State University, U.S.A.), Huey-Liang Huang (National Tsing Hua Univ., Taiwan), C. Schwab (Strasbourg, France).

In this symposium, 52 papers were presented on point defects in compound semiconductors; control and characterization of the stoichiometry of bulk compound semiconductors and the effects of deviations from stoichiometry on their properties; and control, characterization, and effects on device properties of the stoichiometry of surfaces and interfaces.

Dr. Chadi (NEC Research Institute, U.S.A.) reported the results of pseudopotential calculations concerning the bonding environment and electronic structure of point defects in II-VI and III-V compounds. Discussion of the nature of the EL2 trap in GaAs and of the DX center

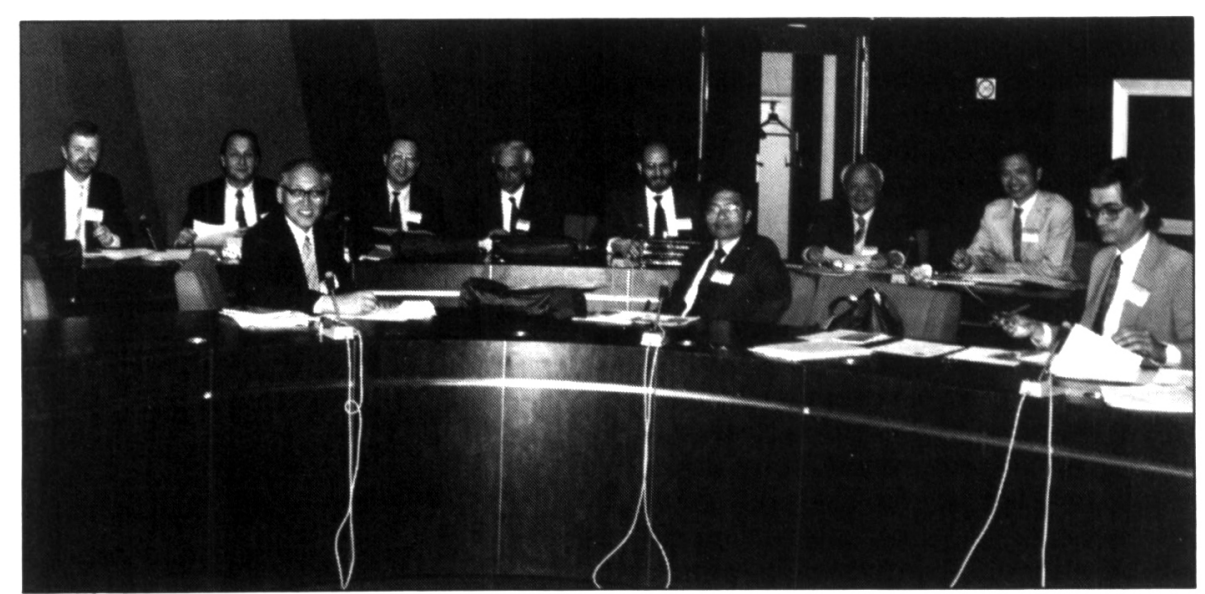

Intemational delegates attend the International Union of Materials Research Societies (IUMRS) Board Meeting in Strasbourg. Front row (left to right): M. Doyama, MRS-Japan; G.C. Chi, MRS-Taiwan; R.C. Ewing, IUMRS secretary. Back row (left to right): I.W. Boyd, E-MRS; P. Siffert, E-MRS; R.P.H. Chang, IUMRS president; G.G. Bentini, E-MRS; E.N. Kaufmann, MRS; C. Shi, C-MRS; and S. Weng, C-MRS. 
started by Dr. Chadi was continued in later sessions on the experimental characterization of point defects. Prof. Spaeth (Univ. of Paderborn, Germany), reported recent results of optically detected magnetic resonance measurements using the magnetic circular dichroism of the optical absorption to identify defects and defect interactions in $\mathrm{GaAs}$ and $\mathrm{GaP}$. He clearly identified the EL2 trap in GaAs as an $A s_{G a}$ antisite pair defect involving the $A s_{i}$ defect. Dr. Gilbart (Centre de Physique de Solides et d'Energie Solaire, France) presented a paper on the DX centers in $\mathrm{Al}_{x} \mathrm{Ga}_{1-x}$ As and $\mathrm{GaP}_{y} \mathrm{As}_{1-y}$ finding that the DX center associated with $\mathrm{Te}$ is located at much higher energy than other donors in the two III-V alloy matrices. Thus Te doping results in less trapping of free electrons, a potential advantage in device manufacturing.

The importance of stoichiometry control to III-V device manufacturing was stressed in the plenary lecture presented by $\mathrm{Dr}$. Oyama on behalf of Prof. Nishizawa of the Semiconductor Research Institute (Sendai, Japan). Dr. Tasumi (Sumitomo Electric Industries, Japan) showed how high quality InP crystals could be produced by a modification of LEC (liquid-encapsulated Czochralski) pulling that utilizes a heated phosphorus vapor-containing region over the encapsulant, thus reducing the tendency toward surface decomposition and thermally induced radial stress. The benefits of understanding the interaction of extrinsic defects with native point defects in III-V compounds were demonstrated by Prof. Pankove (Univ. of Colorado, U.S.A). His studies on the compensation of the nitrogen vacancy donors by substitutional $\mathrm{Zn}$ acceptors in $\mathrm{GaN}$ resulted in realization of a light emitting homodiode in this material with a peak emission of $2.83 \mathrm{eV}$.

A powerful method for detecting both extrinsic and intrinsic point defects in GaAs by measuring $x$-ray quasi-forbidden reflections (XFR) was described by Dr. Fujimoto (Science and Technical Research Laboratories of NHK, Japan). Deviations from stoichiometry of the order of $10^{-4}$ due to an excess of atoms in the As plane were found for LEC-pulled GaAs. The lattice location of $\mathrm{Al}, \mathrm{In}, \mathrm{Si}, \mathrm{Zn}$, and $\mathrm{C}$ and the associated microscopic strain have been determined by XFR measurements. An excellent overview of electrode potential measurements in conjunction with electroanalytical methods for determining activities and concentrations of constituents in compound systems was presented by Prof. Vedel (Ecole Nationale Superieure de Chimie de Paris, France). The usefulness of electroanalytical methods for precise compositional and trace element analyses was demonstrated by Prof. Yang of National Tsing

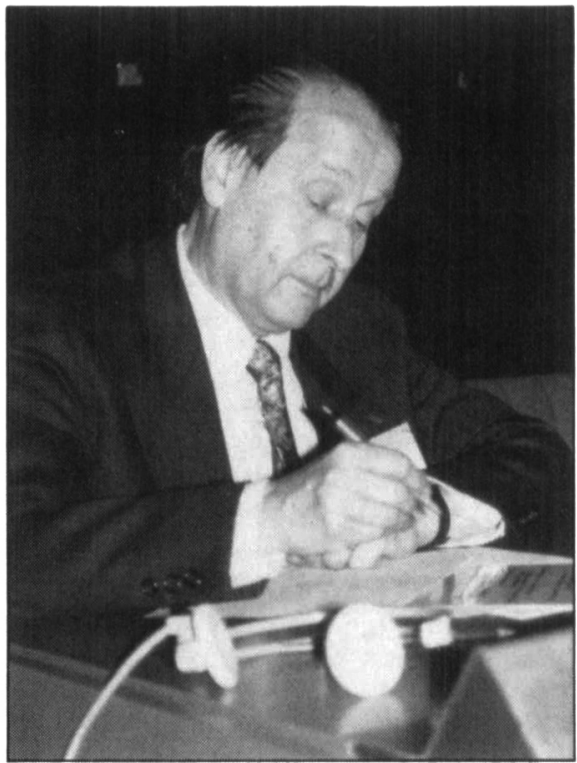

$\mathrm{Ga}_{047} \mathrm{In}_{0.53}$ As layers. $R$. Schedler et al. reported on an ultrathin GaInAs/InP MQW made by interrupted cycle low-pressure metalorganic chemical vapor deposition (LPMOCVD). The presence of a growthinduced $\operatorname{In} P_{0.35} A s_{0.65}$ interlayer at the bottom interface was found to prevent the formation of true type II superlattice LPMOCVD interrupted growth cycles and smooth the interface morphology. Two excellent posters were presented: Dr. Tournie (MaxPlanck-Institut, Stuttgart, Germany) reported on optimizing the growth temperature for the molecular beam epitaxy growth of $\mathrm{Ga}_{0.47} \mathrm{In}_{0.53}$ As on InP; Dr. Chi (Industrial Technology Research Institute, Taiwan) addressed the fabrication of multimode capped mesa-burned-heterostructure $\mathrm{Ga}_{2} \mathrm{In}_{1 \cdot x} \mathrm{P}_{y} \mathrm{As}_{1 \cdot y} / \mathrm{InP}$ laser diodes.

Initial phases of interface formation under chemical beam epitaxy were discussed by Dr. Tsang (AT\&T Bell Laboratories, U.S.A. Although this method has an excellent ability to deposit measured amounts of precursors onto a compound semiconductor surface, even in this favorable case it is difficult to assure complete spreading of the precursors into a uniform monolayer coverage per cycle. The simultaneous control of interface morphology and stoichiometry remains a challenging task. Extraordinary opportunities for designing novel devices and circuits and for studying new electronic interactions in compound semiconductor quantum wells, wires, and boxes were discussed in an inspiring review by Dr. Sahaki (Univ. of Tokyo, Japan). In view of the growing ratio of the interfacial volume to bulk volume with increasing resolution of these heterostructures, one can safely assume that the control of interfacial stoichiometry will be an important aspect of future research in solid state electronics and will promote continuing interest in this topic.

\section{Composite Materials}

Chairs: L. Nicolais (Universita di Napoli, Italy), E. Yasuda (Institute of Technology, Japan), A.T. Di Benedetto (Univ. of Connecticut, U.S.A.).

This symposium covered many significant topics in composite materials, spanning polymer, metal, and ceramic matrix composites. Special sessions were dedicated to composite durability and wear, the physical and mechanical behavior of the interface between matrix and reinforcing agent, and the mechanical and design characteristics of composites. The academic and industrial scientific communities were well-represented at the symposium.

J.C. Halpin (Wright Patterson AFB, 
U.S.A.) gave a perspective of the research in composite materials. He clarified industrial needs in terms of improvement in material properties and processing in order to expand application of composites. Moreover, he clearly explained why a broader use of these materials is limited.

Papers by J.C. Seferis (Univ. of Washington, U.S.A.) and J.M. Kenny (Univ. of Naples, Italy) examined the processing of thermoset-based composite materials in two different cycles of the production cycle. Seferis focused on the impregnation stage of fibers and correlated the preimpregnation characteristics with the quality of the final product. Kenny presented a general approach to the mathematical modeling of composites processing, devoting special attention to autoclave lamination. Starting from kinetic and rheological submodels for the representation of the complex chemorheological behavior of thermoset matrices, he integrated these models with energy balance in order to develop a complete simulation of the process.

L. Nicolais (Univ. of Naples, Italy), analyzed the environmental effects on matrices for high performance composites. $\mathrm{He}$ highlighted the effect of the presence of water and solvents in composite matrices in terms of the change of their physical and mechanical properties. He examined different solvent sorption modes and different forms of water absorbtion in thermosets and thermoplastics matrices.

E. Yasuda (Tokyo Institute of Technology, Japan) investigated the mechanical behavior of $\mathrm{SiC}$ whisker-reinforced alumina matrix composites. He reported the effects of whisker dimension, oxide-layer, and roughness on the fracture toughness of the composite.

K. Friedrich (Institute for Composite Materials, Univ. of Kaiserslautern, Germany) analyzed the wearing behavior of polymer composite materials. He presented the wearing behavior of different types of reinforcement (short and continuous fibers) and different types of matrices (thermosets and thermoplastics) under different wear conditions.

Recipients of the ICAM/E-MRS student award for this symposium presented their papers. N. Han (Univ. of Leeds, England) presented a paper on the interfaces in metal matrix composites reinforced with $\mathrm{SiC}$ particles, and A. Maffezzoli (Univ. of Naples, Italy) presented a paper on using dielectric measurements to characterize the water sorption behavior of epoxybased materials.

The symposium's high scientific quality was reflected in the discussions following most of the presentations. Interest both within the European community and be- yond suggests that E-MRS activity in the composite materials field needs to grow in 1992.

New Materials, Physics and Technologies for Micronic Integrated Sensors

Chair: J.L. Robert (Université de Montpellier II, France).

This three-day symposium, including the E-MRS plenary session, was organized to promote the scientific exchange in research on new materials and the future of modern sensors. The symposium featured 8 invited lectures and 30 papers.

Progress in materials research and recent developments in growth techniques, as well as in technological processes and modeling, have greatly affected advances in sensors. While silicon devices should supply the major need for mechanical sensors, it appears that other materials are opening the way to new applications.

This is the case for $\mathrm{Si}-\mathrm{Ge}$ and $\beta \mathrm{Fe}-\mathrm{Si}_{2}$ films, III-V and II-VI compounds, and structures for optical sensor applications, organic layers (PVDF), high temperature semiconductors ( $\mathrm{SiC}-6 \mathrm{H})$, and onedimensional systems.

Several papers were devoted to studies of surfaces and interfaces, which play a predominant role in the development of sensors.

One-third of the invited papers, talks, and posters were devoted to chemical sensors. Two invited papers concerned upto-date research and new fields of developments in molecular or ionic recognition: "Submicronic Molecular Materials" and "Molecular Materials for the Transduction of Chemical Information into Electrical Signals for Chemfets." The talks of invited speakers, as well as all the other communications, illustrated the importance of the first step toward the development of future chemical sensors.

This symposium revealed that research in this field is very active. The scientific activities of the laboratories in Strasbourg are very complementary. Scientists at the symposium generally agreed that organizing a network of laboratories would be an efficient way to exchange ideas and find common research topics beyond those which occur at regular meetings. In this perspective, the symposium's program committee has suggested creating a network of excellence in the Esprit III Programme.

\section{High Energy Ion Implantation}

Chairs: J. Gyulai (Tech. University Dub. and Central Research Institute for Physics, Hungary), S.U. Campisano (Universita di Catania, Italy).
High energy ion implantation has already found practical applications, mostly in semiconductor device technology. This rather new field is in a stage where research is progressing while industrial applications are lagging.

The symposium began with a joint session with Symposium D, Ion Beam Synthesis of Compound and Elemental Layers. J.M. Poate (AT\&T Bell Laboratories, U.S.A.) showed the broad applications of high energy implantation in materials science, from investigating properties of amorphous silicon to optoelectronics applications. Other invited speakers described the state-of-the-art in specific areas: applications to ULSI (K. Tsukamoto, Mitsubishi, Japan), the role of crystallinity in dopant profiles (V. Raineri, Univ. of Cantania, Italy), the dynamics of lattice damage accumulation (A. Golanski, CNET, France), applications to waveguide lasers ( $P$. Townsend, Univ. of Sussex, U.K.), engineering and other defects (R. Schreutelkamp, FOM, The Netherlands), range parameter measurements, also in 3D (S. Kalbitzer, Max-Planck-Institut, Heidelberg, Germany), high energy and high dose implants effects (F. Paszti, Central Research Institute, Hungary), and implanter characteristics and dopant profile measurements (L. Frey, Frauhofer, Germany); J. Biersack (Berlin, Germany) presented the latest models and calculations of high energy implantation profiles.

Contributed papers covered damage, mixing and phase formation, applications in Si-Ge and compound semiconductors, gettering and lifetime engineering, and four different high energy implantation set-ups including the newly marketed HVEE version of the Tandetron.

Ample time was given to discussion after each presentation, producing interesting debates on future applications of high energy ion implantation. From these discussions the need for more data on particle ranges (in three dimensions) and on the formation of secondary defects in semiconductors emerged. It is believed that this information will fill the gap between near surface and bulk properties of ionirradiated materials.

The symposium proceedings will reach the community directly through Nuclear Instruments and Methods B.

\section{Ion Beam Synthesis of Compound and Elemental Layers}

Chairs: John A. Kilner (Imperial College London, U.K.), Peter L. F. Hemment (University of Surrey, U.K.).

This symposium was one of the first meetings worldwide to be entirely devoted 
to ion beam synthesis (IBS). Europe is particularly well represented in this field. Papers were presented from 13 institutes which are primary producers of ion-beamsynthesized layers, and many more came from collaborating institutes involved in characterizing the synthesized material. At present, most of the community is composed of laboratories involved in IBS of both conducting and insulating layers in silicon; however, at this meeting a small but significant group of papers dealt with nonmicroelectronic topics.

Much of the symposium dealt with the state-of-the-art of the most important ion synthesized material, SIMOX (separation by the implantation of oxygen). These buried layers of silicon dioxide are produced by implanting large doses of oxygen into a silicon wafer. After annealing to produce a stable microstructure with sharply defined layers, the materials are used as silicon-oninsulator substrates for radiation hard and fast MOS circuitry. Key to further development of the technology is the construction of a high throughput, high current implanter capable of processing numerous silicon wafers for commercial-scale production of SIMOX. M. Guerra from IBIS Technology, sponsors of the symposium, showed IBIS's progress in constructing just such a machine. Novel because it has two beam lines going into a single end station, the machine will be able to provide up to 200 $\mathrm{mA}$ current which can be magnetically scanned over the available wafer holders. The machine is designed to process more than 100,000 (6-inch) wafers per year, providing a major step toward SIMOX commercialization.

The production of conducting layers in $\mathrm{Si}$ has also received much attention. A. White (AT\&T Bell Laboratories, U.S.A.) gave an invited talk on the production of epitaxial buried layers of $\mathrm{CoSi}_{2}$ in $\mathrm{Si}$ by the high dose implantation of $\mathrm{Co}$ into $\mathrm{Si}$. One of the most exciting developments was work at Jülich, and several other laboratories, in the production of $\beta-\mathrm{FeSi}_{2}$ layers in $\mathrm{Si}$ by the high dose implantation of $\mathrm{Fe}$ into $\mathrm{Si}$. $\beta-\mathrm{FeSi}_{2}$ is semiconducting and has been reported to have a direct band gap of $0.87 \mathrm{eV}$, of interest for optoelectronic devices, although the band structure of the material is not yet well established. Synthesis of this novel material offers some interesting prospects for device fabrication as well as for the basic materials science of IBS. The Jülich group showed how they can obtain planar epitaxial $\alpha$-FeSi ${ }_{2}$ layers in (111)Si by implantation followed by rapid thermal annealing. These layers can then be converted to $\beta-\mathrm{FeSi}_{2}$ in a further annealing process.
Other topics introduced by invited speakers included the modeling of layer formation, in both the as-implanted and annealed states, and the characterization of the ion-synthesized layers. Overall the symposium reflected the growing interest from all sectors of the materials research community in this novel route for materials production and processing. Plans are to organize a second symposium on ion beam synthesis in two years (1993).

\section{Nuclear Methods in Semiconductor Physics}

Chairs: G. Langouche (Leuven University, Belgium), J.C. Soares (Lisbon University, Portugal), J.P. Stoquert (Centre de Recherches Nucléaires, France).

Contributions in this symposium were largely from two areas of experimental research: (1) the hyperfine interaction methods, focusing on the microscopic configuration surrounding radioactive probe atoms in semiconductors, and (2) ion beam techniques using scattering, energy loss, and channeling properties of highly energetic ions penetrating in semiconductors.

A remarkable observation in the hyperfine interactive research field is the sudden surge of perturbed angular correlation (PAC) studies in the semiconductor field. A nice example of such a reported study is the observation of dynamic effects associated with acceptor-donor pairs in $\mathrm{Si}$, where their local jump motion can be followed by observing the fluctuation in the strength and orientation of the electric field gradient. Radioactive In-probes carefully deposited on ultraclean $\mathrm{Si}$ surfaces led to the first report of surface PAC for semiconductors. Mössbauer spectroscopy (MS), more firmly established in the semiconductor field, was the subject of research topics, drawing attention from different teams: the transition-metal silicides, as $\mathrm{Co}$ and $\mathrm{Fe}$ are excellently suited for MSresearch, and the DX-centers in $\mathrm{Al}_{x} \mathrm{Ga}_{\mathrm{v} \cdot \mathrm{x}} \mathrm{As}$, where $\mathrm{Sn}$ and Te donors are used as MSprobes to unravel the peculiar electronic and structural properties of this defect center. These are just a few examples of local defect studies reported in this symposium. Less commonly used methods in the semiconductor field, such as nuclear magnetic resonance, electron nuclear double resonance, muon spin resonance and positron annihilation, were also reported. A new and original development was derivation of semiconductor density-of-state profiles from high resolution measurements of conversion electron energy spectra.

Emission channeling forms the link be-

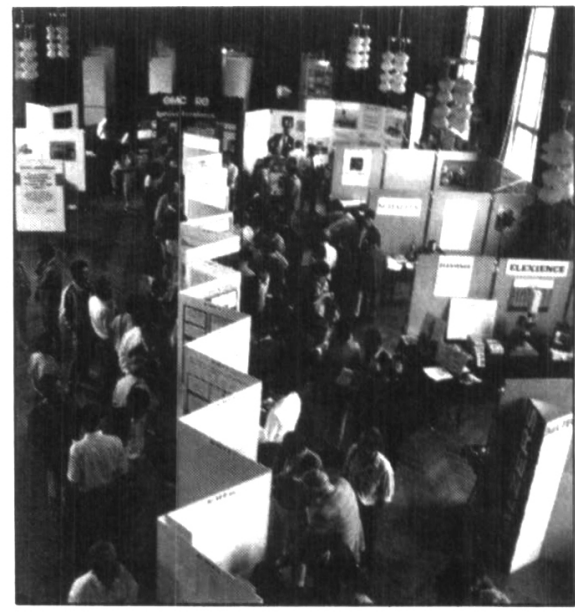

Poster sessions were held in conjunction with the equipment exhibit in the Pavillon Josephine situated in a large park directly across a major bouleward from the Council of Europe facilities.

tween the methods using radioactive probe atoms, and nuclear methods using external beams. Used mainly at the ISOLDE facility in CERN, it has produced an impressive set of lattice location studies with electrons, positrons, and $\alpha$-particles emitted from implanted radioactive atoms.

Among the more exotic reports on ion beam methods were the damage studies after extremely high energy (GeV) heavy ion implantation at the GANIL-facility in Caen, France. Quite interesting studies were also reported using the more conventional Rutherford backscattering, channeling, particle-induced $x$-ray emission, and elastic recoil detection techniques such as the ion implantation defect production in $\mathrm{CdTe}, \mathrm{GaAs}$ and $\mathrm{GaP}$, and a defect analysis for mismatched epitaxial heterostructures. A more fundamental topic was a study of the stopping power for antiprotons in order to derive the importance of the Barkas effect. More application-oriented reports covered neutron transmutation doping and ion beam deposition.

Interweaving hyperfine interaction and ion beam methods in one symposium turned out to be extremely fruitful. Discussions spanned a broad perspective even prompting some researchers to consider alternative models.

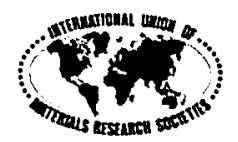

